\section{Community Pharmacy Handbook}

Jon Waterfield (Ed).

Pharmaceutical Press, London, UK.

February 2008.

This book is geared towards pharmacists practicing in the continuously evolving world of community pharmacy. The aim of this book is to provide practical information relevant to community pharmacists and to propose ways of enhancing pharmaceutical care in the community. The Community Pharmacy Handbook covers topics ranging from continuous professional development, to management skills to multidisciplinary collaboration. The versatility of this handbook makes it a useful resource in a variety of different settings. For example, pharmacy managers may seek information related to leading and training a pharmacy team or setting up a service such as a smoking cessation program. Conversely, staff pharmacists may consult this book to learn about responding to patient symptoms or pharmacy students may find it useful to supplement their experiential learning.

The book is divided into 9 chapters. The three introductory chapters address Continuing Professional Development (CPD), management skills and the development of the pharmacy team. The purposes of these chapters are to stress the importance of pharmacists understanding their own professional development, as well as the development and management of their pharmacy team in order to have a successful practice. The next three chapters provide an overview of the new contractual framework for community pharmacy in the United Kingdom (UK), including details on the three categories into which pharmacy services are divided: essential services, additional services such as medicine use reviews (MUR) and enhanced services. Practical tips on setting up additional or enhanced services are also provided. The final three chapters are dedicated to providing information on supplying medications (including a section on pharmacists as prescribers), responding to symptoms and multidisciplinary relationships. The goal of these chapters is to showcase innovative ways to work within pharmacy, as well as with other health care professionals.
Overall, chapters are easy to navigate and are clearly organized into subsections. Each chapter contains the following features:

Checkpoint: Located at the beginning of each chapter, this section contains pre-reading questions which help the reader to identify knowledge gaps, while also providing a brief overview of the main points addressed in the chapter.

Implications for Practice: Appearing at the end of each chapter, this section provides suggested activities which enable the reader to apply key concepts to practice.

Multiple Choice Questions: Each chapter has a set of multiple choice questions for readers to assess their level of knowledge.

Case Studies: A key feature of the handbook is a series of case studies for the reader to integrate knowledge and consolidate key points following each chapter. These case studies are provided in three levels of increasing complexity and are directed at the pharmacy undergraduate student, pre-registration pharmacy graduate (or intern), and community pharmacist.

These features allow readers to assess and integrate their knowledge while applying key concepts to both hypothetical and real-life scenarios.

Overall this book is well written and organized. The author uses examples of common scenarios seen in community practice to explain key concepts and simplify main points. In addition, diagrams, tables, acronyms and practical tips are provided through out each chapter to accommodate various learning styles. Headings and subheadings appropriately organize each chapter. Furthermore, the language is not overly technical, making it a good resource for both pharmacists and students.

This book may not be applicable to pharmacists practicing outside of the UK as there 
are several chapters devoted to the new contractual framework for community pharmacy within the UK. In addition, other chapters include numerous references to the contractual framework, regulations and accreditation standards specific to the UK and are therefore tailored to pharmacists practicing within this jurisdiction.

In addition, this book may not be helpful for the pharmacist requiring an in depth exploration of a particular topic. For example, the chapter on Responding to Symptoms briefly addresses factors to consider when a patient presents to the pharmacy seeking advice. However, it does not provide great detail on communication skills nor patient assessment in specific disease states. The reader may need to consult other resources for this information.

In summary, this handbook is well designed and reasonably priced. It is a good reference for the pharmacist in search of a resource covering a number of topics related to community pharmacy, as well as providing practical tips on how to enhance one's practice. The main limitation is that this book may not be a useful resource for pharmacists practicing outside of the UK.

\section{Andreana Marcinkow}

Pharmacy $4^{\text {th }}$ year undergraduate student

Nese Yuksel B.Sc.Pharm, PharmD

Associate Professor, Faculty of Pharmacy and Pharmaceutical Sciences

University of Alberta

Email: nyuksel@pharmacy.ualberta.ca 